\title{
LATITUDE MANIFESTATIONS OF THE SOLAR WIND
}

\author{
R. VON STEIGER \\ International Space Science Institute \\ Hallerstrasse 6, CH-3012 Bern, Switzerland
}

\begin{abstract}
Dependencies of solar wind parameters as a function of latitude are presented and discussed, based on measurements of the Ulysses mission during times of declining or minimum solar activity. The heliosphere is found to be structured into a band of slow solar wind at low latitudes and two large high-speed streams from the polar coronal holes. Only small trends of solar wind parameters with heliolatitude are found within these strearns. A small but significant temperature difference is seen between the south and the north polar coronal hole.
\end{abstract}

\section{Overview}

The Ulysses mission (Wenzel et al., 1992), launched in 1990, has now almost completed its first revolution on a nearly polar orbit around the Sun. Thus it has mapped the third dimension of the heliosphere for the first time.

An overview of three solar wind parameters, obtained with the SWICS instrument on Ulysses (Gloeckler et al., 1992) is given in Fig. 1: The alpha particle speed, $v_{\alpha}$, the freezing-in temperature obtained from the $\mathrm{O}^{7+} / \mathrm{O}^{6+}$ ratio, $T_{\mathrm{O}}$, and the $\mathrm{Mg} / \mathrm{O}$ abundance ratio.

Evidently, at the current solar minimum conditions, the heliosphere is structured into a band of slow wind around the equator and two high speed streams emanating from the polar coronal holes, with ranges of slow wind alternating with high speed streams at mid-latitudes. The two other parameters, $T_{\mathrm{O}}$ and $\mathrm{Mg} / \mathrm{O}$, are clearly anticorrelated with $v_{\alpha}$, indicating the fundamental difference between these SW types. On the other hand, the fast streams from the coronal holes appear to be very uniform.

In the following sections we will discuss three types of latitude manifestations as observed by SWICS/Ulysses:

- The large asymmetry between the two quasistationary solar wind types, slow wind from above the streamer belt and fast streams from coronal holes.

- Trends of solar wind parameters as a function of heliographic latitude within the (largely structure-free) high-speed streams.

- Differences between the solar wind parameters from the north and the south polar coronal holes.

\section{Low/High Latitude Asymmetry}

In Fig. 2 (from Geiss $e t$ al., 1995), we illustrate the difference between the slow wind from above the streamer belt and the fast streams from coronal holes. The data were obtained with SWICS/Ulysses in 1992-93, when the two stream types were alternating once per solar rotation due to the fact that the center of the polar coronal hole was offset from the solar rotation axis. Nine such recurrences were superposed and an average profile was obtained for each parameter considered in order to enhance the statistics.

The anticorrelation of the freezing in temperatures and of both the $\mathrm{Mg} / \mathrm{O}$ and the $\mathrm{Fe} / \mathrm{O}$ abundance ratios are particularly evident in this representation. Since these parameters probe the conditions and processes at the source regions of the solar wind, this indicates that the slow wind and the fast streams are fundamentally different: 


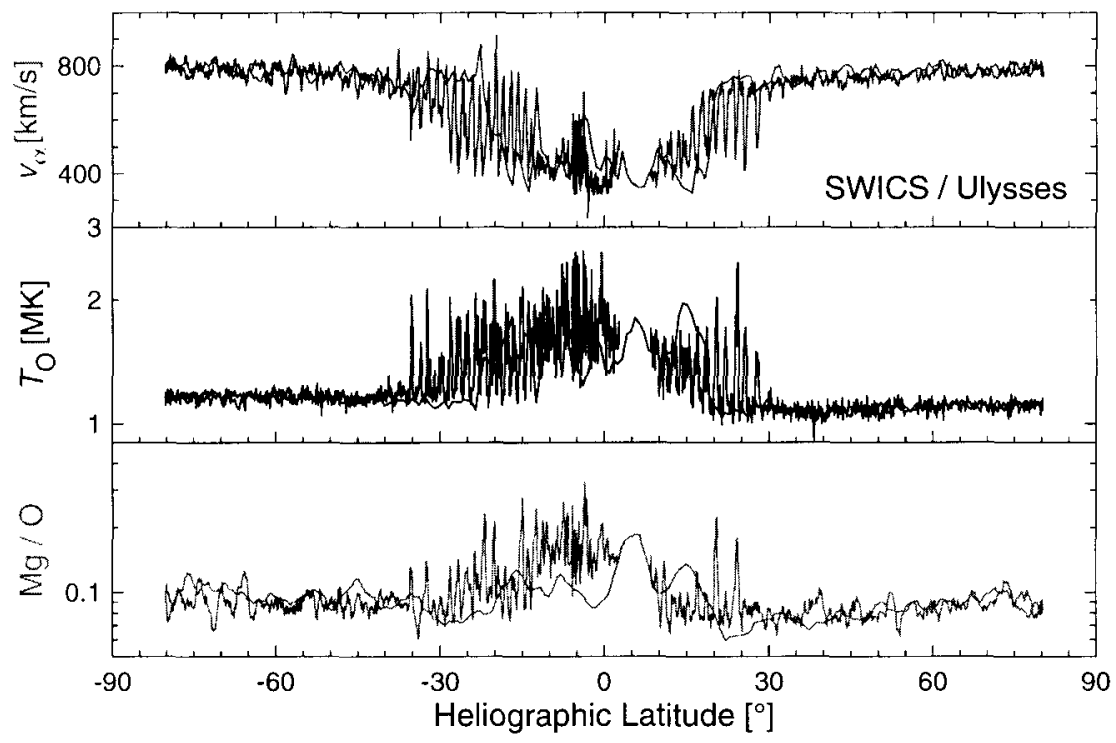

Figure 1 . Overview of three solar wind parameters obtained with SWICS/Ulysses (top: speed of alpha particles, $v_{\alpha}$; middle: freezing-in temperature obtained from the $\mathrm{O}^{7+} / \mathrm{O}^{6+}$ ratio, $T_{\mathrm{O}}$; bottom: $\mathrm{Mg} / \mathrm{O}$ abundance ratio) as a function of heliographic latitude. Daily average values are given from launch to date; the curves start in October 1990 at about $+3^{\circ}$, reach $-80^{\circ}$ in September 1994 and, after the fast latitude scan, $+80^{\circ}$ in August 1995 , and end in July 1997 at about $+8^{\circ}$. The shaded band indicates the latitude range of the Earth's orbit. All three parameters show an obvious difference between the solar wind from low and from high latitudes.

(1) The freezing-in temperatures from the heavy ion charge state ratios are consistently lower in the fast streams (Fig. 2, upper panel). Since they probe the electron temperature in the coronn at 1.5 to $3.5 R_{\odot}$ (depending on the ratio considered), this confirms that the coronal hole is cooler than the streamer belt. Moreover, for each element a single freezing-in temperature may be found in the fast streams, allowing to derive a temperature profile with altitude (Geiss $e t(a l ., 1995)$, but not in the slow wind, indicating that this solar wind type might be mixed from thermally different reservoirs.

(2) The abundances of heavy ions with a low first ionization potential (FIP) such as $\mathrm{Mg}$ or Fe are enhanced relative to the high-FIP ions such as $\mathrm{O}$ by a factor of 3-5 in the slow wind as compared to the photosphere (Fig. 2, lower panel). This FIP effect most likely operates by atom-ion separation in the chromosphere and thus probes the conditions at this layer (Geiss, 1982). The fact that it is much weaker, if not altogether absent (enhancement factor 1-2), in the fast streams indicates a difference in chromospheric conditions beneath coronal holes as compared to closed field regions (von Steiger $6 t$ al., 1997).

The fact that the freezing-in temperatures and and the strength of the FIP effect change in unison (cf. Fig. 2) implies that the two stream types are separated by a boundary extending through the corona down into the chromosphere. Recently, Wimmer-Schweingruber ot al. (1997) have shown that the change of both these compositional signatures occurs exactly at the stream interface within the corotating interaction regions. This in turn implies that the two solar wind types maintain a well-preserved identity at least out to $\sim 5 \mathrm{AU}$.

It should also be noted that the fast streams at the time of these observations extended down to $\sim 20^{\circ}$ in latitude, whereas the coronal hole boundaries were located around $\sim 60^{\circ}$, indicating that the polar streams expand superradially by about a factor of five in solid angle.

\section{Variations Inside Coronal Holes}

The fast streams represent a remarkably uniform, structureless solar wind type (Bame $\epsilon t a l ., 1977$ ) of almost photospheric elemental composition (von Steiger et al., 1997, and references therein). 


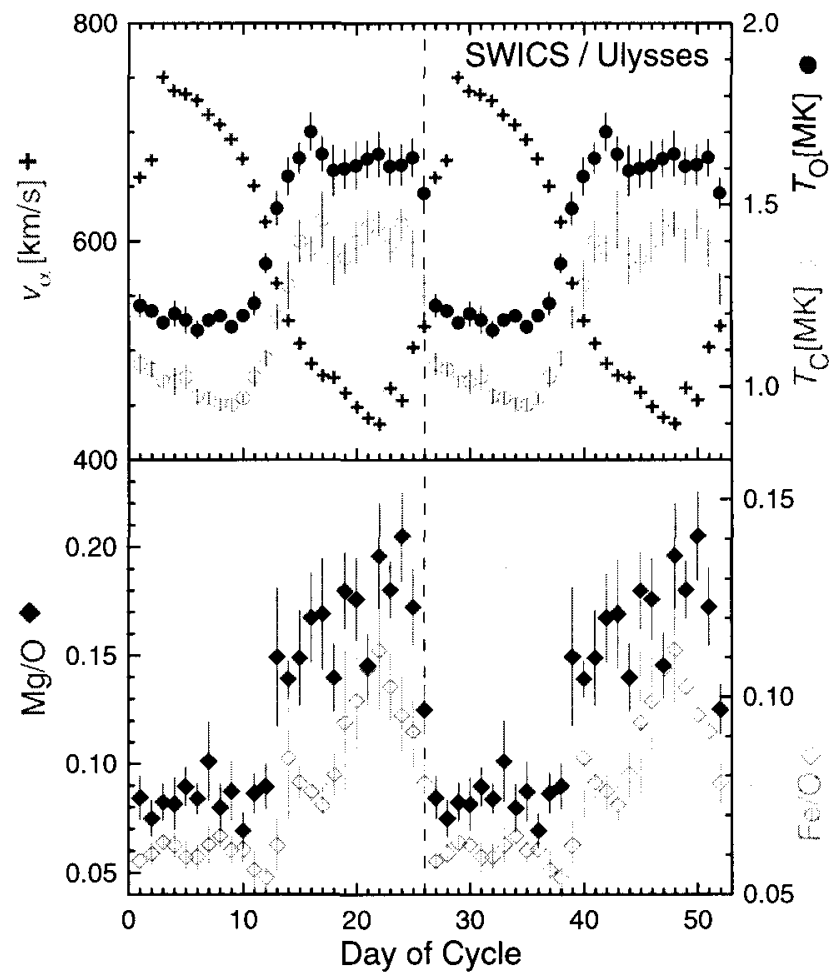

Figure 2. Superposed epoch representation of nine periods of alternating slow wind and high-speed streams, observed in 1992-93. The freezing-in temperatures, $T_{O}$ and $T_{C}$ (top panel), as well as the the strength of the FIP bias, i.e. the $\mathrm{Mg} / \mathrm{O}$ and $\mathrm{Fe} / \mathrm{O}$ ratios (bottom panel), are clearly anticorrelated with the solar wind speed, with remarkably sharp boundaries between the two stream types. The data are repeated twice in order to bring out the pattern more clearly. (Figure from Geiss et al., 1995.)

In Fig. 3 we show the solar wind speed and the freezing-in temperature as a function of latitude when Ulysses was completely immersed in the north or south polar stream. Both polar streams look remarkably similar in $v_{\alpha}(\phi)$, and there is a small but distinctive trend of about $1 \mathrm{~km} / \mathrm{s}$ per degree latitude, with the highest speed occurring near the center of the polar coronal holes. [For comparison, the latitudinal speed gradient between slow wind and fast streams varied from 14 to $28 \mathrm{~km} / \mathrm{s}$ per degree during the fast latitude scan of Ulysses in 1995 (Neugebauer et al., 1997).] The converse is true, however, for the freezing-in temperatures, the trend of which with latitude is hardly significant. Yet there is an obvious difference in $T_{0}$ observed between the north and the south polar hole, which will be discussed in the next section.

As is the case for the charge states (or freezing-in temperatures), there seems to be no significant trend of the elemental ratios such as $\mathrm{Mg} / \mathrm{O}$ with latitude within the coronal holes, and no significant difference of $\mathrm{Mg} / \mathrm{O}$ between the $\mathrm{N}$ and $\mathrm{S}$ hole could be found either. The question whether the variations seen in $\mathrm{Mg} / \mathrm{O}$ within the polar holes (cf. Fig. 1) are significant and perhaps might be associated with a microstructure of the holes such as the one reported by Neugebauer et al. (1995) will be addressed in a forthcoming paper.

\section{North/South Coronal Hole (A)Symmetry}

The freezing-in temperature $T_{\mathrm{O}}$ in Fig. 3 (right) shows a clear difference between the $\mathrm{N}$ and $\mathrm{S}$ polar coronal hole of $\sim 0.1 \mathrm{MK}$, with the $\mathrm{N}$ hole being the cooler one. This difference, first reported by Ipavich et al. (1995), is also seen in the charge state distributions of $\mathrm{C}, \mathrm{Mg}, \mathrm{Si}$, and $\mathrm{Fe}$. As is the case in the south hole (Geiss et al., 1995), the charge state distributions of $\mathrm{Si}^{7+}$ to $\mathrm{Si}^{11+}$ and of 

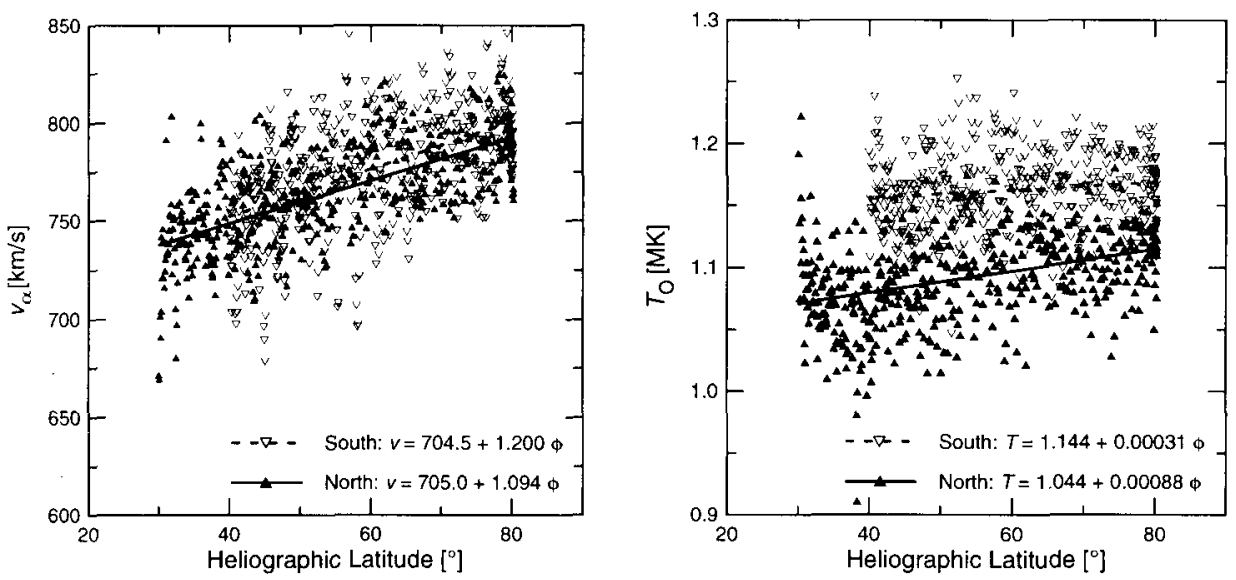

Figure 3. Daily average values of $v_{\alpha}$ (left) and of $T_{0}$ (right) vs. latitude $\phi$, measured by SWICS/Ulysses inside the polar high-speed streams southward of $-40^{\circ}$ and northward of $+30^{\circ}$, respectively. A weak but significant trend, but no difference between the $\mathrm{N}$ and the $\mathrm{S}$ stream, is observed in $v_{\alpha}(\phi)$, while the opposite is true for $T_{O}(\phi)$.

$\mathrm{Fe}^{7+}$ to $\mathrm{Fe}^{13+}$ may also be fitted by a single temperature in the north as well, but they are shifted towards lower charge states ( $0.6 \mathrm{MK}$ for $\mathrm{Si}$ and $0.8 \mathrm{MK}$ for $\mathrm{Fe}$, respectively), indicating a lower electron temperature there, consistent with the $T_{\mathrm{O}}$ observations.

Note that the observations in the $\mathrm{N}$ and $\mathrm{S}$ fast streams were taken at different times separated by at least four months. It is therefore possible that the two holes have the same temperature, but were cooling down between the south and the north polar pass of Ulysses. However, this seems to be a less likely possibility than a true spatial effect because little or no temporal trend was observed when traversing the individual holes.

In the slow wind, the charge state distributions of the heavy elements are wider, $\mathrm{Si}^{7+}$ to $\mathrm{Si}^{12+}$ and $\mathrm{Fe}^{6+}$ to $\mathrm{Fe}^{16+}$, respectively, and they do not reflect a single temperature. This indicates that the slow wind traversed a complicated thermal profile on its way through the corona, or that it is a mixture of different reservoirs such as closed loops of varying temperatures opening up by reconnection with open field lines near the coronal hole boundaries (Schwadron et al, 1997)

\section{Summary}

There is a large and clear difference between the slow solar wind at low latitudes and the fast streams from the polar coronal holes; it is most pronounced in the speed, the freezing-in temperatures, and the strength of the FIP effect.

The fast streams from coronal holes are very uniform; only a small dependency of the speed, but no significant dependencies of the other parameters with latitude were found.

A small asymmetry between the north and the south polar stream was found in the freezing-in temperatures, indicating that the north polar coronal hole was cooler by up to $0.1 \mathrm{MK}$.

\section{Acknowledgements}

I gratefully acknowledge many useful discussions with J. Geiss and G. Gloeckler and with many members of the SWICS team and of the Ulysses project.

\section{References}

Bame, S. J., Asbridge, J. R., Feldman, W. C., and Gosling, J. T.: 1977, J. Geophys. Res. 82, 1487-1492.

Geiss, J.: 1982, Space Sci. Rev. 33, 201-217.

Geiss, J., Gloeckler, G., von Steiger, R., et al: 1995, Science 268, 1033-1036.

Gloeckler, G., Geiss, J., Balsiger, H., et al:: 1992, Astron. A strophys. Suppl. 92, 267-289.

Ipavich, F. M., Cohen, C. M. S., Gloeckler, G., Galvin, A. B., Ko, Y.-K., Geiss, J., Woch, J., and von Steiger, R.: 1995, Eos Trans. AGU 76(46), F452. 
Neugebauer, M., Forsyth, R. J., Galvin, A. B., Harvey, K. L., Lazarus, A. J., Lepping, R. P., Linker, J., Mikic, Z., Steinberg, J. T., von Steiger, R., Wang, Y.-M., and Wimmer-Schweingruber, R.: 1997, J. Geophys. Res., submitted.

Neugebauer, M., Goldstein, B. E., McComas, D. J., Suess, S. T. and Balogh, A.: 1995, J. Geophys. Res. 100, 23,38923,395 .

Schwadron, N. A., Fisk, L. A., and Zurbuchen, T. H.: 1997, Astrophys. J., submitted.

von Steiger, R., Geiss, J. and Gloeckler, G.: 1997, in J. R. Jokipii, C. P. Sonett, and M. S. Giampapa (eds), Cosmic Winds and the Heliosphere, University of Arizona Press, Tucson, in press.

Wenzel, K.-P., Marsden, R. G., Page, D. E., and Smith, E. J.: 1992, A stron. Astrophys. 92, 207-219.

Wimmer-Schweingruber, R. F., von Steiger, R., and Paerli, R.: 1997, J. Geophys. Res, 102, 17,407-17,417. 